\title{
TOWARDS SVC-BASED ADAPTIVE STREAMING IN INFORMATION CENTRIC NETWORKS
}

\author{
Stefano Petrangeli, Niels Bouten, Maxim Claeys and Filip De Turck \\ Department of Information Technology (INTEC), Ghent University-iMinds \\ Gaston Crommenlaan 8 (Box 201), 9050 Ghent, Belgium, email: stefano.petrangeli@intec.ugent.be
}

\begin{abstract}
HTTP Adaptive Streaming (HAS) is becoming the de-facto standard for video streaming services. In HAS, each video is segmented and stored in different qualities. The client can dynamically select the most appropriate quality level to download, allowing it to adapt to varying network conditions. As the Internet was not designed to deliver such applications, optimal support for multimedia delivery is still missing. Information Centric Networking (ICN) is a recently proposed disruptive architecture that could solve this issue, where the focus is given to the content rather than to end-to-end connectivity. Due to the bandwidth unpredictability typical of ICN, standard AVC-based HAS performs quality selection sub-optimally, thus leading to a poor Quality of Experience (QoE). In this article, we propose to overcome this inefficiency by using Scalable Video Coding (SVC) instead. We individuate the main advantages of SVC-based HAS over ICN and outline, both theoretically and via simulation, the research challenges to be addressed to optimize the delivered QoE.
\end{abstract}

Index Terms - Adaptive Streaming, Scalable Video Coding, Quality of Experience, Information Centric Networking

\section{INTRODUCTION}

Over the past decades the Internet traffic caused by multimedia services such as video streaming has increased considerably and is projected to exceed 90 percent of all Internet traffic by 2017 [1]. Video streaming puts a huge strain on the underlying delivery network. Historically, the Internet has evolved in an ad-hoc manner were incremental patches were added to handle new requirements as they arose. This means that the underlying network model has not changed over the last decades, while the services that use the Internet have changed drastically. This prohibits the optimal sup-

Niels Bouten and Maxim Claeys are funded by a Ph.D. grant of the Agency for Innovation by Science and Technology in Flanders (IWT). This work was partly funded by Flamingo, a Network of Excellence project (318488) supported by the European Commission under its Seventh Framework Programme.

978-1-4799-7082-7/15/\$31.00 (c)2015 IEEE port for current multimedia-driven network delivery. These shortcomings have motivated academia to propose alternative architectures. Information Centric Networking (ICN) is among these revolutionary architectures, which moves the traditional focus of a host-oriented communication model to a content-centric model. To accomplish this, ICN relies on location-independent naming schemes, in-network caching and content-based routing to allow an efficient distribution of content over the network.

The majority of the aforementioned multimedia traffic comes from streaming services such as Netflix and Hulu. In contrast to managed Internet Protocol television (IPTV) services, these Over-The-Top (OTT) services leverage standard HTTP infrastructure to deliver the video. To cope with heterogeneous and dynamically changing network conditions, these services are based on HTTP Adaptive Streaming (HAS) technologies. This allows a scalable delivery of the video by shifting the rate adaptation towards the client. To achieve this, the video is split temporally into chunks which are called segments and is encoded at different quality rates. The adaptation heuristic residing at the client selects which quality rate should be downloaded for each segment. Using buffer filling levels, network statistics and device characteristics, the client is able to respond to throughput fluctuations by seamlessly switching to a lower quality representation.

Traditionally, Advanced Video Coding (AVC) is used in HAS to encode the individual segments. Recently, a new standard called High Efficiency Video Coding (HEVC) has also been proposed. Since in AVC each quality layer is independent of all other quality layers, this introduces a significant amount of redundancy, both in storage and delivery of the segments. Scalable Video Coding (SVC) can cope with these issues by creating dependencies between the different quality layers, allowing an increased efficiency in both storage, caching and delivery of the segments. This allows the rate adaptation heuristic to gradually upgrade the quality, avoiding the negative impact of wrong quality decisions. This is not the case for AVC based HAS clients, since once a quality decision is made, the segment cannot be upgraded to a higher quality, nor can a partially received segment be decoded at a lower quality. This causes AVC-based adaptation heuristics to be strongly dependent on throughput estimations to decide 
which quality to download. Overestimations of the available bandwidth can quickly drain the buffer and lead to playout interruption. Especially in an ICN context, where segments can be served through various caches with different throughput, a bandwidth-estimation based adaptation can lead to bad Quality of Experience (QoE).

Each of the above-mentioned technologies have some unique advantages when deployed separately, combining all three of them yields additional advantages. When considering a live TV scenario, the camera to display delay should be as low as possible. However, in traditional HAS deployments, a large play-out buffer is required to guarantee a continuous playout. Furthermore, since HAS over IP uses unicast connections, large amounts of redundant traffic is introduced when multiple clients stream the same content. By combining HAS with SVC, the incremental character of SVC allows to shrink the buffer since the base layer guarantees a continuous playout. Adding ICN to the mix allows a multicast-like distribution of the content through the ICN-nodes, which, together with the lower encoding redundancy of SVC, allows to drastically reduce the traffic redundancy for live TV streaming.

This paper focuses on combining together the advantages of HAS, SVC and ICN. Furthermore, the challenges that arise from their combination are identified and several mitigation techniques are proposed to overcome these challenges.

\section{RELATED WORK}

Nowadays, HAS has become more prominent than ever. Several large industrial players, including Microsoft (Silverlight Smooth Streaming), Apple (HTTP Live Streaming) and Adobe (HTTP Dynamic Streaming) have commercial HAS implementations. MPEG, in collaboration with other standard groups, such as 3GPP, standardized the HAS interfaces and protocol data in Dynamic Adaptive Streaming over HTTP (DASH) in 2011 [2], establishing a common ground between the vast amount of available implementations.

The H.264/AVC codec is most commonly used for HAS video. For each quality representation, the source video has to be encoded into an independent AVC video. This obviously leads to a significant amount of redundant data, stored at the server. This is alleviated by the introduction of the SVC extension of AVC [3]. Huysegems et al. describe the advantages and disadvantages of SVC-based HAS [4]. The authors show that using SVC, the storage and bandwidth requirements are significantly reduced while a theoretically better play-out is achieved in scenarios with fluctuating bandwidth conditions. This, however, comes at the cost of a significant overhead in terms of segment bitrate for the higher quality layers, introduced by the nature of SVC encoding. Furthermore, as the different quality layers have to be downloaded separately, SVC is more vulnerable to high round trip times. Bouten et al. show that this can be mitigated by using HTTP pipelining or parallel downloads [5]. Famaey et al. show that AVC performs better under high latencies, while SVC is bet- ter suited to adapt to temporary bandwidth fluctuations when using a small buffer [6]. The benefits of using SVC in terms of caching and uplink bandwidth are discussed by Sanchez et al. $[7,8]$.

Liu et al. propose to parallelize the download and requests of HAS segments to achieve a better resource utilization in 3GPP networks [9]. The bandwidth consumption can also be decreased by grouping unicast HAS sessions, sharing the same content, into a single multicast session, as shown by Bouten et al. [10]. A SVC-specific quality selection heuristic is presented by Andelin et al., to take into account more specific algorithmic decisions, possible for SVC. In this algorithm, the trade-off between downloading the next segment and upgrading a previously download segment is defined using a slope [11].

Lederer et al. identify the possibilities of applying HAS over ICN and present an architecture for DASH over CCN [12]. Furthermore, several open challenges are identified by the authors. The name-based routing in ICN causes the origin server to be transparent to the HAS clients. This leads to unwanted behaviour as bandwidth measurements, considered in most adaptation heuristics, are no longer valid when switching between origin servers. A similar problem is introduced by the seamless handover between multiple interfaces, supported in ICN. Yu et al. propose a DASH-aware scheduling algorithm for edge cache prefetching to improve the QoE of video streaming in ICN, by utilizing residual bandwidth to request video segments in advance [13].

Even though ICN and SVC-based adaptive streaming have separately received some attention, no research to date has tackled the combined problem yet. In order to overcome this issue, we analyze in this paper the gains brought by SVC over ICN and the challenges that still need to be addressed to optimize the delivered QoE.

\section{SVC-BASED ADAPTIVE STREAMING OVER ICN}

In this section, we provide a comprehensive analysis of the advantages and challenges deriving from the adoption of SVC adaptive streaming in ICN. The use of standard AVC-based HAS in ICN comes with a number of drawbacks. First, the bandwidth unpredictability typical of ICN environments, makes it difficult for the client to perform an efficient adaptation. Particularly, this leads to an increased number of quality switches and video freezes, two of the main factors influencing users' QoE. Due to its layered structure, SVC adaptive streaming is inherently more robust to video freezes compared to AVC and can consequently perform better in ICN environments. Second, AVC HAS does not allow to fully exploit one of the main innovations introduced by ICN, i.e., the possibility to seamlessly use all the available network interfaces of a device. This especially represents an advantage in mobile networks, where the video streaming client is equipped with different antennas, such as Wi-Fi and 3G/4G. In standard AVC HAS, only the interface providing the high- 
est bit-rate is used to forward an interest (i.e., the requested segment at a certain quality level). The layered structure of SVC provides a natural way to use multiple interfaces simultaneously, thus increasing the aggregated throughput for the client and, consequently, optimizing the resulting QoE.

In the authors' vision, the use of SVC in ICN networks would allow to solve the aforementioned inefficiencies and exploit the full potential of both technologies, as detailed in the remainder of this section.

\subsection{Opportunities}

The use of SVC adaptive streaming in ICN provides a natural synergy to both increase users' QoE and network efficiency. Particularly, we identify three main advantages deriving from the combined use of SVC-based HAS and ICN, which we thoroughly describe in the following.

\subsubsection{Application to live video streaming}

According to the general ICN concept, when a node receives an interest for a content not stored in the local cache, the interest is forwarded to a neighboring node. Further interests for the same content are not forwarded, but added to the so-called Pending Interest Table (PIT). When the content is retrieved by the node, all the pending interests contained in the PIT table are satisfied. Due to this inherent support for multicast, ICN is particularly suited for live video streaming scenarios. From a QoE perspective, an important aspect to consider in live streaming is the minimization of the camera-to-display delay, which is the delay between recording an event and its playout on the client's display. In current HAS deployments, this delay is in the order of tens of seconds, because a large buffer at the client is generally used to prevent play-out freezes. An SVC-based client is especially suited in this scenario, as the layered structure of the video allows to use a smaller buffer compared to AVC. In light of the above, SVC represents the best technology for live streaming in ICN networks, due to its capability to adapt to bandwidth conditions and minimize the camera-to-display delay.

\subsubsection{Leveraging Multiple Interface Utilization}

One of the main advantages brought by ICN, is the possibility to seamlessly use all the available network interfaces of a device to forward an interest. In current adaptive streaming deployments though, a single interface is used to forward an interest, namely the one providing the highest throughput. The layered structure of SVC opens a wide range of optimizations to solve this inefficiency. While in AVC each segment at different quality represents an interest, we foresee that in SVC each quality layer composing a single playable segment will represent a separate interest. This would allow the client to request different SVC layers in parallel over different interfaces, in order to increase the aggregate throughput.

A fundamental aspect of this solution is represented by the interface selection for the request of the different SVC

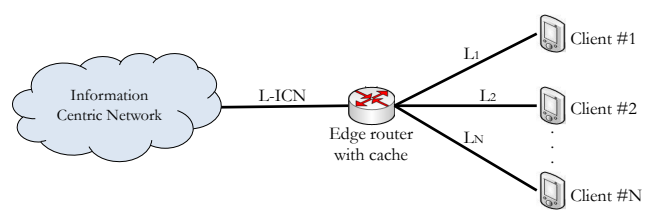

Fig. 1: Schematic representation of a communication network with $N$ clients streaming video.

layers. Based on the bandwidth perceived on the available interfaces, the client can schedule the download of the different layers in order to: (i) guarantee a timely download of the base layer, thus avoiding a video freeze and (ii) maximize video quality by downloading as much enhancement layers as possible. Several download strategies can be envisioned in this case. For example, the base layer can be requested over the interface providing the highest throughput, or it can be downloaded in parallel over all the available interfaces in order to minimize the risk of freezes.

\subsubsection{Impact on caching and resource utilization}

The use of SVC in combination with ICN also has positive effects in terms of caching and network efficiency. In ICN, each node is equipped with the so-called content store (CS), where the content can be cached. This entails that, theoretically, every node in the network can provide caching functionalities. By using SVC adaptive streaming, the cache hit rates for lower layers are increased compared to AVC, because lower layers always have to be requested by the client in order to play-out a segment. Consequently, this increases the chances for lower layers to be kept in the cache. This would represent an advantage over AVC, especially in Video-on-Demand (VOD) scenarios. Moreover, a higher caching efficiency allows to lower the bandwidth usage in ICN networks, as lower layers are cached (even if clients select different quality levels) and, consequently, do not need to be continuously transmitted among the nodes. The same considerations are not valid for AVC adaptive streaming, as segments at different quality levels are completely independent from each other.

For completeness, we provide a calculation of the bandwidth usage in ICN when SVC or AVC adaptive streaming is used. We consider a schematic network architecture where an edge router with caching functionalities is connected to an ICN network via link $L-I C N$ and to a set of $N$ video streaming clients, as depicted in Figure 1. We assume the clients are streaming the same content (e.g., a live event) and the number of clients to be greater than the number of available quality levels of the video, indicated by $Q L$. In the worst case scenario, all clients will request each segment of the video at different quality levels. The bandwidth usage per segment on link $L-I C N$ strongly depends on the use of AVC or SVC, and can be quantified as in the following:

$$
\begin{aligned}
B W_{A V C} & =\sum_{i=1}^{Q L} \text { BitRate }_{A V C}(i) \\
B W_{S V C} & =\text { BitRate }_{S V C}(Q L)
\end{aligned}
$$




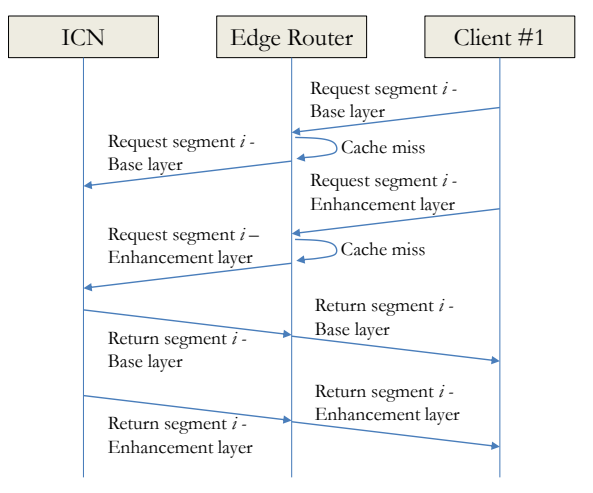

(a)

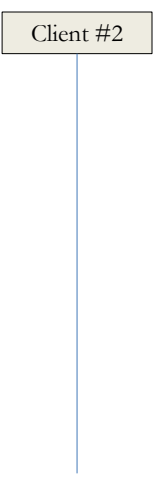

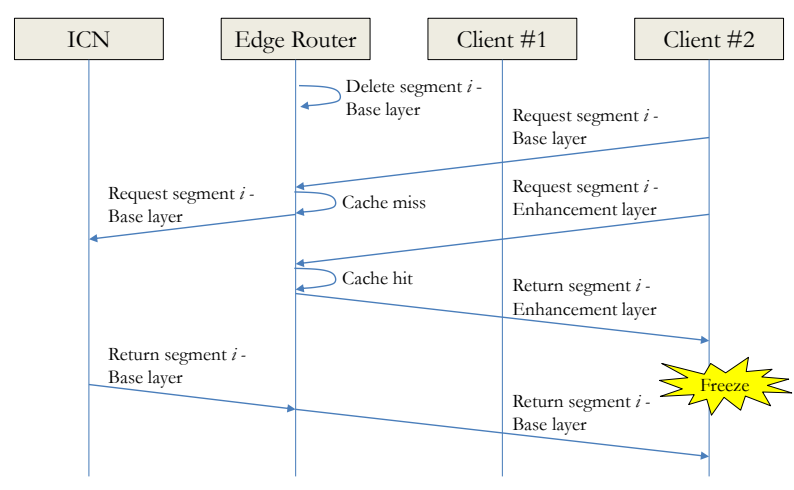

(b)

Fig. 2: An example of the negative effects caused by cache misses for the base layer of SVC-based HAS.

where $\operatorname{BitRate}_{A V C}(i)$ represents the bit rate of the $i$-th quality level for AVC, while $\operatorname{BitRate~}_{S V C}(i)$ represents the cumulative bit-rate in the SVC case. In AVC, all the different quality levels have to be separately downloaded from the ICN network before they can be cached in the router. The bandwidth consumed on link $L-I C N$ is thus equal to the sum of the bit-rates of all the available quality levels (Eq. 1). On the contrary, in SVC, all the quality levels can be served by using the layers cached during the download of the highest quality level. Consequently, the bandwidth consumed on $L-I C N$ is only equal to the cumulative bit-rate of the highest available quality (Eq. 2).

\subsection{Research Challenges}

As explained in the previous section, several advantages arise when SVC is used in combination with ICN. The aim of this section is to provide an overview of the challenges that still need to be addressed and to outline some possible solutions to mitigate them.

\subsubsection{Diffused quality representations}

The main characteristic of SVC adaptive streaming is the inter-dependency between the different layers composing a playable segment. This means that the base layer always has to be available in order to avoid a freeze and decode the enhancement layers. In this perspective, cache misses for the lower layers are particularly detrimental, as this increases the segment download time and can likely lead to buffer starvations. As an example, we consider the scenario as in Figure 1, with two clients streaming the same video (i.e., $N=2$ ). We also assume the edge router to be equipped with a Least Recently Used (LRU) cache. As shown in Figure 2a, Client \#1 requests segment $i$ at quality level $q l$, with $q l$ greater than 1 . As the edge cache is empty, both the base and enhancement layers have to be downloaded from the ICN network, cached and delivered to Client \#1. If segment $i$ is not requested for a specific period of time, the LRU cache starts to remove the base layers first, as they are requested and delivered before the enhancement layers (see Figure 2b). This way, if Client \#2 requests segment $i$ at the same quality level $q l$, the enhancement layers can be downloaded from the cache, while the base layers, necessary in order to play the enhancement layers, have still to be retrieved from the ICN network. This procedure increases the risk of freezes, especially if the bandwidth on link $L-I C N$ drops.

A possible mitigation strategy to this problem is represented by SVC-aware ICN caches. In this case, the ICN node is aware of the inter-dependency between the different quality layers of SVC videos. Consequently, more efficient caching rules can be specified, where a specific quality layer is only removed if higher quality layers are not present in the cache. In the previous example, the edge node would remove the enhancement layers first, thus allowing Client \#2 to retrieve the base layers directly from the cache, preventing a freeze.

\subsubsection{Bandwidth (un)predictability}

One of the main disadvantages coming from the adoption of adaptive streaming techniques in ICN, is represented by the reduced predictability of future bandwidth conditions. As the client is not aware of the node that is actually delivering the segments, a reliable bandwidth estimation cannot be performed. Particularly, this happens when the bottleneck is not located in the access network (e.g., the radio interface) but is actually located in the ICN network itself. A natural mitigation to this problem is inherently provided by the use of SVC, as the client can still play the base layer if a wrong estimation was made on the future throughput. Nevertheless, even a SVC client can experience video freezes if the bandwidth is highly unpredictable.

A possible solution to bandwidth unpredictability is represented by purely buffer-based (BB) rate adaptation heuristics. In BB heuristics, the client makes the decision on the quality level to download on the basis of the current buffer filling level only, without considering the perceived bandwidth. For SVC, downloading the base layer first allows to reduce the risk of freezes and quickly ramp-up the buffer. The video 


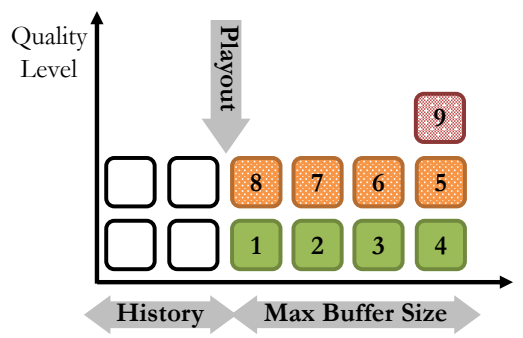

Fig. 3: Buffer filling strategy for SVC-based HAS

quality of the downloaded segments can still be incremented once the buffer filling is sufficiently high, thanks to the incremental quality encoding typical of SVC. In order to accelerate these operations, the client can make use of multiple network interfaces at the same time, as presented previously. A similar approach for AVC-based HAS would lead to an increased overhead and a lower quality.

\section{EXPERIMENTAL STUDY}

To evaluate the impact of both AVC and SVC-based HAS over ICN, an NS- $3^{1}$ based network simulator was used in combination with the Network Simulation Cradle ${ }^{2}$. For AVCbased HAS, we implemented a client based on an implementation of Microsoft Smooth Streaming as described in [6]. A simple buffer-based heuristic was used to implement the rate adaptation for the SVC-based client. The rate adaptation always starts by checking the buffer for the presence of base layer segments. If not all base layer segments are available, the base layer of the segment with the earliest playout time is downloaded. If all base layer segments are present, the rate adaptation starts upgrading the quality from right to left as shown in Figure 3, by first downloading the enhancement layer of the segment with the latest playout time. This simple rate adaptation strategy ensures that the base layer segments are downloaded first, before starting to upgrade the quality.

One of the challenges introduced by HAS over ICN is that the client is not aware of the actual source of the content, introducing challenges for adaptation heuristics based on the estimated bandwidth. To demonstrate the negative effects introduced by this issue, we performed simulations where the segments are served from different ICN nodes at different rates. The rates for these nodes were uniformly distributed between $0.5 \mathrm{Mbps}$ and $5 \mathrm{Mbps}$. We encoded and segmented a video for both AVC and SVC at 3 quality rates using a segment length of $2 s$. The resulting layer bitrates are [1042kbps, 1390kbps, 1958kbps] and [1059kbps, 484kbps, 902kbps] for AVC and SVC respectively. In all the experiments, a live video streaming scenario has been evaluated. To avoid the impact of the encoding overhead of SVC on the graphs, we show the played quality level instead of the playout bitrates.

\footnotetext{
${ }^{1}$ http: //www.nsnam.org

${ }^{2}$ http://research. wand.net.nz/software/nsc.php
}

Figure 4(a) shows the impact of the fluctuating segment download rates on the playout quality level for both AVC and SVC using a buffer of $12 s$. The buffer-based adaptation of SVC is able to guarantee a continuous playout, completely avoiding buffer starvations even though the per segment download rate is fluctuating heavily as shown in Figure 4(c). On the contrary, AVC MSS uses both the buffer filling and throughput estimations when selecting the quality to download. Since the throughput is unpredictable, the AVC MSS heuristic often overestimates the available bandwidth and downloads a too high quality. This leads to very long download times, depleting the buffer and leading to frame freezes. Figure 4(b) shows the buffer starvation length for each segment, illustrating that the wrong bandwidth estimations can lead to buffer starvations of up to $8 s$ and yield a total freeze time of $48.6 s$, heavily impacting the QoE.

To evaluate the impact of different buffer sizes, we run a series of simulations with varying buffer sizes. Each of these experiments was repeated 10 times and the graphs show both the average values and $95 \%$ confidence intervals. Figure 5(a) shows that for SVC, the playout quality level is a bit lower compared to AVC. This can be accounted to the encoding overhead of SVC, requiring more bits to be transferred to achieve the same quality level, as well as to the more conservative quality improvement behaviour of SVC. Figure 5(b) shows the total buffer starvation length for both AVC and SVC. These results show that a buffer of only 2 segments is too small to cope with the highly varying bandwidth, leading to almost $40 \mathrm{~s}$ of buffer starvations for both approaches. When the buffer size increases, the total starvations for AVCbased HAS remain around $32 s$, while for SVC, they drop to 1.5s. As Figure 5(c) shows, SVC buffer-based rate adaptation is also able to maintain a more stable quality, i.e., less quality switches take place.

\section{CONCLUSIONS}

In this paper, we presented an overview of the advantages and challenges deriving from the adoption of SVC in ICN environments. Classical AVC videos are not able to fully exploit the potential of ICN. Particularly, they can lead to suboptimal adaptation due to the bandwidth unpredictability typical of ICN, thus leading to unnecessary quality switches or video freezes. Moreover, they do not exploit the inherent capability of ICN of using multiple interfaces at the same time. On the contrary, SVC represents a natural solution in this case, due to its layered structure. This means that different layers can be requested at the same time over multiple interfaces, thus increasing the overall throughput and users' QoE. We also elaborated on how SVC can increase the efficiency of ICN-based networks and can be used in ICN to provide the best $\mathrm{QoE}$ in live video streaming scenarios. Some major challenges were also identified, in terms of bandwidth predictability and cache misses. Future research will include the numerical evaluation 


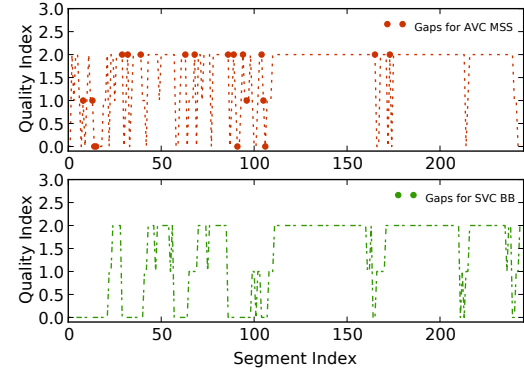

(a) Played quality level for each segment

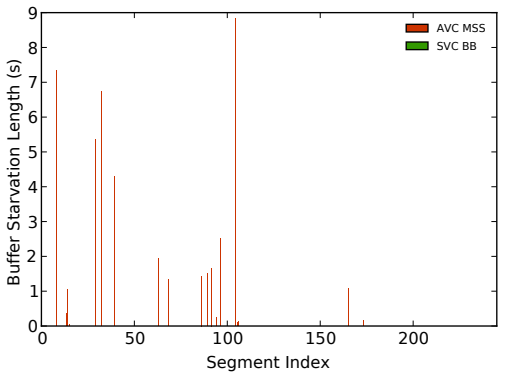

(b) Buffer starvation time for each segment (s)

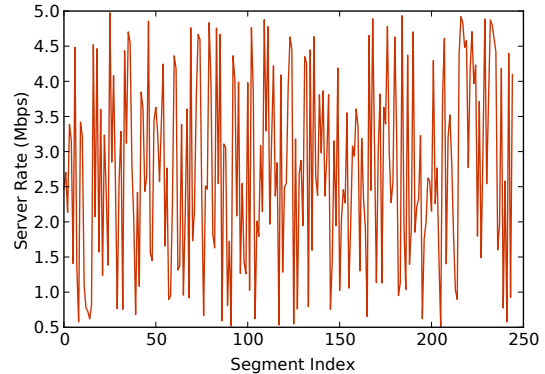

(c) Throughput for each segment (Mbps)

Fig. 4: Impact of varying server rate for a single run using a buffer of $12 \mathrm{~s}$

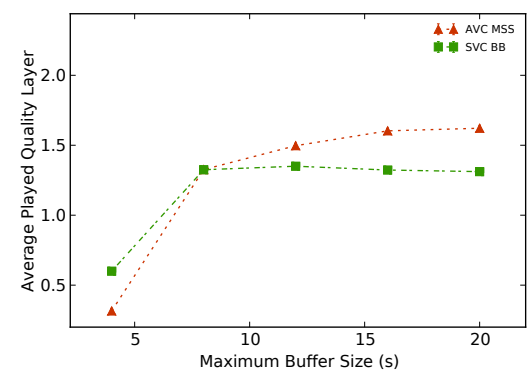

(a) Impact on average quality level

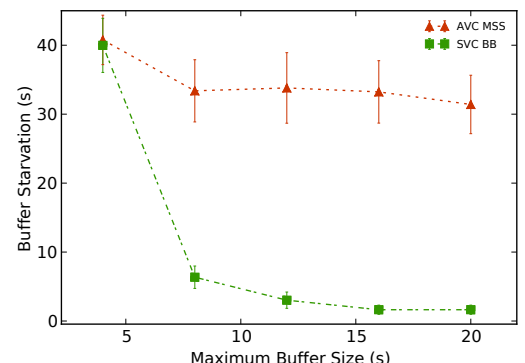

(b) Impact on total buffer starvation time (s)

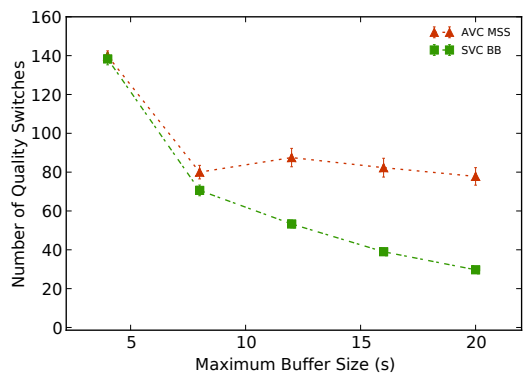

(c) Impact on average number of switches

Fig. 5: Impact of buffer size for both AVC and SVC based streaming.

of the advantages obtained by using SVC over ICN, both from a client- and network-perspective, by using the ICN-enabled DASH client developed by Lederer et al. [12].

\section{REFERENCES}

[1] Cisco VNI Forecast, "Cisco visual networking index: Global mobile data traffic forecast update 2012-2017," Tech. Rep., Cisco Public Information, May 2013.

[2] T. Stockhammer, "Dynamic adaptive streaming over HTTP: standards and design principles," in ACM Conference on Multimedia Systems (MMSys), 2011.

[3] H. Schwarz, D. Marpe, and T. Wiegand, "Overview of the scalable video coding extension of the h.264/avc standard," Circuits and Systems for Video Technology, IEEE Transactions on, vol. 17, no. 9, pp. 1103-1120, Sept 2007.

[4] R. Huysegems, B. De Vleeschauwer, T. Wu, and W. Van Leekwijck, "Svc-based http adaptive streaming," Bell Lab. Tech. J., vol. 16, no. 4, pp. 25-41, Mar. 2012.

[5] N. Bouten, S. Latré, J. Famaey, F. De Turck, and W. Van Leekwijck, "Minimizing the impact of delay on live svc-based http adaptive streaming services," in Integrated Network Management (IM 2013), 2013 IFIP/IEEE International Symposium on, May 2013, pp. 1399-1404.

[6] J. Famaey, S. Latré, N. Bouten, W. Van de Meerssche, B. De Vleeschauwer, W. Van Leekwijck, and F. De Turck, "On the merits of svc-based http adaptive streaming," in Integrated Network Management (IM 2013), 2013 IFIP/IEEE International Symposium on, May 2013, pp. 419-426.

[7] Y. Sanchez, T. Schierl, C. Hellge, T. Wiegand, D. Hong, D. De Vleeschauwer, W. Van Leekwijck, and Y. Le Louedec, "idash: Improved dynamic adaptive streaming over http using scalable video coding," in Proceedings of the Second Annual ACM Conference on Multimedia Systems, New York, NY, USA, 2011, MMSys '11, pp. 257-264, ACM.

[8] Y. Sanchez, T. Schierl, C. Hellge, T. Wiegand, D. Hong, D. De Vleeschauwer, W. Van Leekwijck, and Y. Le Louedec, "Efficient http-based streaming using scalable video coding," Signal Processing: Image Communication, vol. 27, no. 4, pp. 329 342, 2012.

[9] I. Chenghao L., Bouazizi and M. Gabbouj, "Parallel adaptive http media streaming," in Computer Communications and Networks (ICCCN), 2011 Proceedings of 20th International Conference on, July 2011, pp. 1-6.

[10] N. Bouten, S. Latré, W. Van de Meerssche, B. De Vleeschauwer, K. De Schepper, W. Van Leekwijck, and F. De Turck, "A multicast-enabled delivery framework for qoe assurance of over-the-top services in multimedia access networks," Journal of Network and Systems Management, vol. 21, no. 4, pp. 677-706, 2013.

[11] T. Andelin, V. Chetty, D. Harbaugh, S. Warnick, and D. Zappala, "Quality selection for dynamic adaptive streaming over http with scalable video coding," in Proceedings of the $3 \mathrm{rd}$ Multimedia Systems Conference, New York, NY, USA, 2012, MMSys '12, pp. 149-154, ACM.

[12] S. Lederer, C. Mueller, C. Timmerer, and H. Hellwagner, "Adaptive multimedia streaming in information-centric networks," Network, IEEE, vol. 28, no. 6, pp. 91-96, Nov 2014.

[13] Y. Yu, F. Bronzino, R. Fan, C. Westphal, and M. Gerla, "Congestion-aware edge caching for adaptive video streaming in information-centric networks," in IEEE Consumer Communications \& Networking Conference (CCNC), 2015. 